\title{
Análisis DAFO de las actitudes y percepciones del profesorado y alumnado de inglés como lengua extranjera hacia el aprendizaje de idiomas asistido por ordenador: estudio intercultural en Irán y España
}

\author{
Dara Tafazoli, Cristina A. Huertas Abril \& $M^{a}$ Elena Gómez Parra \\ Universidad de Córdoba \\ z52tatad@uco.es, cristina.huertas@uco.es, elena.gomez@uco.es \\ https://dx.doi.org.10.12795/futhark.2018.il3.10
}

Fecha de recepción: 13.2.2018

Fecha de aceptación: 15.5.2018

Resumen: Este estudio aborda el diseño de una matriz DAFO basada en las actitudes y percepciones de profesorado y alumnado de idiomas en Irán y España para explorar las fortalezas, oportunidades, debilidades y amenazas del Aprendizaje de Lenguas Asistido por Ordenador (conocido como CALL, acrónimo de Computer-Assisted Language Learning), así como las diferencias existentes entre estos dos contextos. Los datos recopilados se analizaron mediante análisis estadístico (que incluyó el análisis del contenido de los datos cualitativos) para clasificar los datos y alinearlos en la matriz DAFO. Las conclusiones de este trabajo apuntan a las implicaciones pedagógicas, así como a una serie de recomendaciones para seguir investigando en este campo.

Palabras clave: Aprendizaje de Lenguas Asistido por Ordenador, CALL, estudio intercultural, análisis DAFO.

\section{A SWOT Analysis of the Attitudes and Perceptions of EFL Teachers and Students towards a computer-assisted Language Learning: an intercultural Analysis in Iran and Spain}

Abstract: This study addresses the design of a SWOT matrix based on language teachers' and students' attitudes and perceptions in Iran and Spain to explore the strengths, opportunities, weaknesses, and threats of Computer-Assisted Language 
Learning (CALL), as well as the existent differences between these two contexts. Collected data were scrutinized through statistical analysis (which included content analysis of qualitative data) to classify data and align them into the SWOT matrix. Our conclusion points to pedagogical implications as well as recommendations for further research.

Keywords: Computer-Assisted Language Learning, CALL, Cross-cultural study, SWOT analysis.

Sumario: I. Introducción. 2. Metodología. 2.I. Participantes. 2.2. Instrumento. 2.3. Diseño de la investigación. 2.4. Análisis de datos. 3. Resultados. 3.I. Matriz DAFO de las percepciones y actitudes de profesorado hacia CALL. 3.2. Matriz DAFO de las percepciones y actitudes del alumnado hacia CALL. 3.3. Matriz DAFO de las similitudes en percepciones y actitudes de profesorado y alumnado. 4. Discusión y conclusión.

\section{Introducción}

Son numerosos los autores que indican que se deben combinar diversos elementos para un uso exitoso de la tecnología en el proceso de enseñanzaaprendizaje de lenguas extranjeras (Hubbard y Levy, 2006; Kassen, Lavine, MurphyJudy, y Peters, 2007; Kessler, 2010; Son, Robb, \& Charismiadji, 20II). En este contexto, destacan las investigaciones sobre las ventajas y desventajas del aprendizaje de lenguas asistido por ordenador (más conocido por sus siglas en inglés, CALL, que corresponden a Computer-Assisted Language Learning). No cabe duda de que en la actualidad el software, las herramientas y los programas de CALL están ampliamente disponibles para el profesorado y alumnado de lenguas a nivel mundial (aunque esta accesibilidad puede variar dependiendo del país). A pesar de las diversas facetas positivas de CALL, todavía puede desempeñar un papel funcional en el desarrollo de competencias lingüísticas, pues habitualmente se incide en la (falta de) disponibilidad de recursos CALL como justificación habitual para su implementación limitada (Tafazoli, 2015).

Un análisis del estado de la cuestión nos indica que el uso de CALL ha sido explorado desde diferentes perspectivas. Así, una serie de investigaciones han estudiado las competencias de los profesores en la implementación de CALL y han descubierto por qué algunas herramientas y programas de CALL son más habituales que otros (Golshan y Tafazoli, 20I4; Hubbard y Levy, 2006; Mumtaz, 2000; Son, Robb, y Charismiadji, 20I I). Además, diversos estudios han investigado la forma en que los profesores implementan herramientas CALL en sus aulas (Eubanks, Yeh, y Tseng, 2018; Henry, Carroll, Cunliffe, y Kop, 2018; Jin, 2018; Schulze y Scholz, 2018; Yang, 2018). Aunque muchos estudiosos abordan las actitudes de los profesores hacia CALL (véase, por ejemplo, Lam, 2000), la mayoría 
de los análisis previos sobre las actitudes hacia CALL se sitúan en una cultura y contexto particulares.

Por otra parte, son numerosos los estudios que han investigado las actitudes de los estudiantes hacia CALL (p. ej. Hamid, Waycott, Kurnia, y Chang, 2015; Heflin, Shewmaker, y Nguyen, 2017; Horvat, Dobrotaa, Krsmanovic, y Cudanova, 2013; Kung, 2018; Lin, Warschauer, y Blake, 2016; Lintunen, Mutta, y Pelttari, 2017; Ozdamli y Uzunboylu, 2015; Pinto-Llorente, Sánchez-Gómez, García-Peñalvo, y Casillas-Martín, 2016; Riemer y Schrader, 2015; Wright, 2017). En España, PintoLlorente et al. (2016) analizaron las actitudes positivas hacia la tecnología de una muestra de alumnado español, destacando lo siguiente: (I) fomento del aprendizaje autónomo e individualizado, (2) entorno natural y real (exposición auténtica) y materiales auténticos para la práctica de la lengua, (3) desarrollo del aprendizaje colaborativo, (4) flexibilidad, (5) aumento de la motivación de los estudiantes, y (6) posibilidad de realizar una autoevaluación continua. Asimismo, podemos destacar que Hamid et al. (2015) llevaron a cabo un estudio intercultural sobre las percepciones de los estudiantes malayos y australianos respecto al uso de las tecnologías sociales con el fin de mejorar las interacciones en el aprendizaje de idiomas, donde destacaron los siguientes aspectos clave: (I) mayor implicación con respecto al contenido, (2) mejora en el aprendizaje entre iguales, (3) desarrollo del pensamiento crítico, (4) fomento del aprendizaje autónomo, (5) seguimiento individualizado del progreso del aprendizaje, (6) interacción con el profesorado, y (7) entorno de aprendizaje agradable e interactivo.

A pesar de todo lo anterior, ningún análisis ha abordado cualitativamente las actitudes y percepciones del profesorado y del alumnado de lenguas extranjeras en entornos culturales diferentes, a pesar de que los estudios interculturales son un modo efectivo de explorar los rasgos psicológicos (Matsumoto y Yoo, 2006) que pueden proporcionar una mejora educativa (Stigler y Hiebert, 1999). A tenor de lo expuesto anteriormente, $y$ teniendo en cuenta la importancia de los estudios interculturales en el ámbito educativo, este trabajo plantea la siguiente pregunta de investigación: ¿Cuáles son las fortalezas, debilidades, oportunidades y amenazas de CALL para el proceso de enseñanza-aprendizaje de lenguas extranjeras de acuerdo con las percepciones y actitudes de docentes y estudiantes iraníes y españoles?

Para responder a esta pregunta, tras esta introducción, se presenta la metodología, prestando especial atención a los procedimientos de recolección de datos cualitativos y a la realización de la matriz DAFO (acrónimo correspondiente a Debilidades, Amenazas, Fortalezas y Oportunidades), incluyendo tres matrices (docentes, alumnado y comparativa profesorado-alumnado) en la sección de resultados. Finalmente, en el último apartado, se discuten las implicaciones y las limitaciones del estudio. Hemos de destacar que, en este trabajo, el aprendizaje de idiomas asistido por ordenador (CALL) se entiende como cualquier aplicación de la 
tecnología para la enseñanza y el aprendizaje de idiomas (Tafazoli, Gómez, y Huertas, 2018).

\section{Metodología}

\section{I. Participantes}

Este estudio se realizó en el curso 2017-2018 en Irán y España, siendo la población meta el profesorado y el alumnado de lenguas extranjeras. La muestra total contaba con 318 profesores y 307 estudiantes de lenguas extranjeras. Se pidió a los participantes de ambos grupos que respondieran a un total de diez preguntas abiertas de forma voluntaria, para lo cual se utilizó un cuestionario en línea creado en Google Forms. Finalmente, 277 de los 318 docentes y 237 de los estudiantes proporcionaron respuestas válidas para el estudio.

Entre los participantes del grupo de profesorado, el 50,9\% eran docentes iraníes, mientras que el $49,1 \%$ eran españoles, como se ilustra en la Tabla I. Además, las mujeres eran el género dominante en este grupo (64,6\%), mientras que 98 de los 277 participantes de la muestra eran varones (35,4\%).

Tabla I. Distribución de los participantes del grupo de profesorado de inglés como lengua extranjera de acuerdo con su nacionalidad y género

\begin{tabular}{ccc}
\hline \multirow{2}{*}{ País } & Género & $\begin{array}{c}\text { N. }{ }^{\circ} \\
\text { Participantes }\end{array}$ \\
\hline \multirow{3}{*}{ Irán } & Masculino & 58 \\
& Femenino & 83 \\
& Total & 141 \\
\cline { 2 - 3 } España & Masculino & 40 \\
& Femenino & 96 \\
\cline { 2 - 3 } Total & Total & 136 \\
\cline { 2 - 3 } & Masculino & 98 \\
& Femenino & 179 \\
& Total & 277
\end{tabular}

Con respecto al grupo del alumnado, el $62,9 \%$ eran iraníes y el $37,1 \%$ españoles, como se muestra en la Tabla 2 . Además, las mujeres fueron nuevamente el género dominante (75, I \%), mientras que 59 de los 237 (24,9\%) participantes de la muestra eran hombres. 
Tabla 2. Distribución de los participantes del grupo de estudiantes de inglés como lengua extranjera de acuerdo con su nacionalidad y género

\begin{tabular}{ccc}
\hline \multirow{2}{*}{ País } & Género & $\begin{array}{c}\text { N. } \\
\text { Participantes }\end{array}$ \\
\hline \multirow{3}{*}{ Irán } & Masculino & 33 \\
& Femenino & 116 \\
\cline { 2 - 3 } España & Total & 149 \\
\cline { 2 - 3 } & Masculino & 26 \\
& Femenino & 62 \\
\cline { 2 - 3 } Total & Total & 88 \\
\cline { 2 - 3 } & Masculino & 98 \\
& Femenino & 178 \\
& Total & 237 \\
\hline
\end{tabular}

En la Tabla 3 se recoge la distribución de los profesores de acuerdo con su titulación: Licenciatura/Grado, Máster y Doctorado. El grupo minoritario en términos de nivel educativo fue Licenciatura/Grado, con un total de 44 participantes, mientras que el grupo principal fue el de docentes con Máster, con I5I participantes.

Tabla 3. Distribución de los participantes del grupo de profesorado de inglés como lengua extranjera de acuerdo con su nivel educativo

\begin{tabular}{ccc}
\hline \multirow{3}{*}{ País } & Titulación & N. ${ }^{\circ}$ Participantes \\
& & \\
\hline \multirow{3}{*}{ Irán } & Licenciatura/Grado & 24 \\
& Máster & 78 \\
& Doctorado & 39 \\
\cline { 2 - 3 } España & Licenciatura/Grado & 20 \\
& Máster & 73 \\
\cline { 2 - 3 } Total & Doctorado & 43 \\
\cline { 2 - 3 } & Licenciatura/Grado & 44 \\
& Máster & 151 \\
& Doctorado & 82 \\
\hline
\end{tabular}

Fuente: Elaboración propia 
De igual modo, la distribución de las titulaciones del grupo de estudiantes tampoco fue uniforme en la muestra (véase Tabla 4). El grupo minoritario en términos de nivel educativo fue el de estudiantes de lengua con Doctorado, que sumaron 3I participantes, mientras que el grupo principal correspondía con Licenciatura/Grado, con 107 respuestas.

Tabla 4. Distribución de los participantes del grupo de estudiantes de inglés como lengua extranjera de acuerdo con su nivel educativo

\begin{tabular}{ccc}
\hline \multirow{3}{*}{ País } & Titulación & N. ${ }^{\circ}$ Participantes \\
& & \\
\hline \multirow{3}{*}{ Irán } & Licenciatura/Grado & 73 \\
& Máster & 59 \\
& Doctorado & 17 \\
\cline { 2 - 3 } España & Licenciatura/Grado & 34 \\
& Máster & 40 \\
\cline { 2 - 3 } Total & Doctorado & 14 \\
\cline { 2 - 3 } & Licenciatura/Grado & 107 \\
& Máster & 99 \\
\hline & Doctorado & 31 \\
\hline
\end{tabular}

\subsection{Instrumento}

Para validar el instrumento, los investigadores decidieron aplicar la metodología Delphi, de manera que el cuestionario inicial para esta investigación fue diseñado y enviado a veinte doctores expertos en las siguientes áreas Lingüística Aplicada, Ciencias de la Computación, Enseñanza del Inglés como Lengua Extranjera y CALL, y procedentes de diferentes partes del mundo como Irán, España, Estados Unidos y Reino Unido, entre otros.

La fase de recolección y análisis de datos del estudio Delphi estuvo guiada por tres aspectos clave: el descubrimiento de opiniones, el proceso de determinación de los temas más importantes y la gestión de los comentarios (Keeney, Hasson, y McKenna, 2000). En primer lugar, los investigadores trataron de descubrir las opiniones de los expertos para llegar a un consenso sobre el contenido del cuestionario. En segundo lugar, después de recoger las opiniones de los expertos, los datos se analizaron mediante la técnica de análisis de contenido. Por último, y tras tres rondas de análisis y consenso, los investigadores acordaron la versión final del cuestionario. 
Tras este proceso, finalmente el cuestionario estuvo compuesto por 14 preguntas de información demográfica y 10 preguntas abiertas. La recogida de datos en forma de cuestionario es uno de los métodos más habituales para analizar percepciones y opiniones en una investigación a gran escala (Mackey, y Gass, 2005). En este sentido, Phellas, Bloch, y Seale (20II) señalan algunas ventajas de los cuestionarios en línea:

(1) Web page surveys are extremely fast. (2) No cost is involved once the setup has been completed. (3) You can show pictures, video and play sound. (4) Web page questionnaires can be set with skip instructions. (5) Web page questionnaires can use colours, fonts and other formatting options not possible in most email surveys. (6) A significant number of people will give more honest answers to questions. (7) People give longer answers to openended questions. (8) Survey answers can be combined with pre-existing information you have about individuals taking a survey. (p. 190)

Los cuestionarios en línea proporcionan la entrada y codificación automática de datos así como su edición y evaluación; de igual modo, los participantes en este estudio pudieron acceder de manera autónoma y flexible a través de los enlaces en línea proporcionados.

\subsection{Diseño de la investigación}

Este estudio cualitativo incluyó preguntas abiertas para conocer las percepciones de profesorado y alumnado de inglés como lengua extranjera de Irán y de España hacia CALL. El análisis DAFO de los datos recolectados se realizó manualmente, si bien el análisis estadístico incluyó el análisis del contenido de los datos cualitativos para clasificarlos y alinearlos en la matriz DAFO. En el presente estudio se aplicó el análisis de contenido, una técnica de investigación para hacer inferencias replicables y válidas de los datos a su contexto (Krippendorf, 1980) que emplea un conjunto de procedimientos para hacer inferencias válidas a partir del texto (Weber, 1990).

\subsection{Análisis de datos}

El análisis DAFO busca identificar las debilidades, amenazas, fortalezas y oportunidades de un fenómeno, a fin de analizar los factores que intervienen en él para mejorar y predecir las posibles dificultades, buscando la planificación estratégica a corto y a largo plazo (Thamrin y Pamungkas, 2017).

En este estudio se aplicaron varios pasos para realizar el análisis DAFO, a saber: (a) recopilación de información, (b) categorización de los datos en 
debilidades, amenazas, fortalezas y oportunidades, (c) determinación del valor de cada factor, y (d) presentación del resultado en una matriz.

En este estudio, los investigadores construyeron un sistema basado en cuestionarios para automatizar el proceso de análisis DAFO (ver Figura I), de modo que la matriz se elaboró a partir de las respuestas al cuestionario de los participantes de dos países (Irán y España). La respuesta a cada una de las preguntas abiertas del cuestionario fue de carácter cualitativo, de modo que necesitaron un procesamiento de texto en forma de análisis de contenido para ayudar a determinar si la respuesta era de carácter positivo o negativo.

\section{Diseño y desarrollo de un cuestionario}

\section{Formulación de un sistema de evaluación de las}

\section{respuestas}

Respuestas cualitativas de los participantes

Procesamiento de texto en forma de análisis de contenido y categorización de los datos

\section{Matriz DAFO}

Figura I. Proceso de análisis de datos. Fuente: Elaboración propia.

Las respuestas cualitativas fueron procesadas mediante el análisis de contenido antes de ser clasificadas en la matriz DAFO. Los investigadores aplicaron el análisis de contenido para categorizar los datos de texto en respuestas positivas - negativas, tras lo cual se calculó la ponderación de las puntuaciones y se categorizó el contenido. Posteriormente, se clasificaron los factores en categorías DAFO de acuerdo con el contenido de los datos cualitativos. Los investigadores adoptaron el Modelo de Reglas (Thamrin y Pamungkas, 2017) para categorizar los factores basados en las respuestas de los participantes (ver Tabla 5). 
Tabla 5. Modelo de reglas (basado en Thamrin y Pamungkas, 2017)

\begin{tabular}{cccc}
\hline & & \multicolumn{2}{c}{ Valoración } \\
\cline { 3 - 4 } Factor & Interno & Positivo & Negativo \\
\cline { 3 - 4 } & Externo & Oportaleza & Debilidad \\
& \multicolumn{2}{c}{ Fuente: Elaboración propia. } & Amenaza \\
\hline
\end{tabular}

El Modelo de Reglas discrimina las respuestas basadas en factores externos e internos. Posteriormente, los investigadores categorizaron las respuestas basándose en sus puntuaciones positivas o negativas, clasificándose estas directamente en función de su contenido para la realización de la matriz DAFO.

\section{Resultados}

Antes de analizar los resultados, los investigadores quieren señalar que la matriz DAFO de este estudio fue diseñada desde el punto de vista del profesorado y del alumnado $y$, por tanto, puede ser muy diferente de otros grupos (por ejemplo, administradores). En primer lugar, se presenta la matriz DAFO de los resultados del profesorado; a continuación, se recoge la matriz DAFO de los resultados del alumnado; finalmente, se presenta una matriz conjunta con aspectos clave que comparten ambos grupos.

Por lo tanto, los factores internos son los que tienen que ver con los profesores, mientras que los factores externos tienen que ver con los estudiantes y la propia tecnología.

\section{I. Matriz DAFO de las percepciones y actitudes del profesorado hacia CALL}

En esta matriz, los factores internos están relacionados con el profesorado, mientras que los factores externos tienen que ver con el alumnado y la propia tecnología.

Tabla 6. Matriz DAFO del profesorado de lenguas extranjeras de Irán y de España (por frecuencia de respuesta).

\begin{tabular}{|c|c|}
\hline Fortalezas & Debilidades \\
\hline $\begin{array}{l}\text { - La enseñanza mediante CALL es } \\
\text { cómoda y fácil de usar (ES/IR) } \\
\text { - Enseñar con CALL es divertido } \\
(E S / I R)\end{array}$ & $\begin{array}{l}\text { - Existe una falta de competencia } \\
\text { digital / en el uso de CALL por } \\
\text { parte de los docentes (ES/IR) } \\
\text { - CALL permite un menor control } \\
\text { del profesor sobre los alumnos }\end{array}$ \\
\hline
\end{tabular}


- CALL facilita que el entorno, los materiales y la comunicación sean ricos y auténticos (ES/IR)

- Ahorro de tiempo (ES/IR)

- CALL aumenta la creatividad de los docentes (ES/IR)

- CALL mantiene a los maestros actualizados (IR)

- CALL permite un mayor control en el aula a través de CALL (ES/IR)

- $\quad C A L L$ es útil (ES/IR)

- CALL permite la enseñanza y el aprendizaje personalizado / individualizado (ES/IR)

- Se puede utilizar en cualquier momento y lugar (ES/IR)

- CALL mejora la colaboración e interacción entre pares (ES)

- CALL aumenta la calidad de la enseñanza (IR)

- CALL sirve para cubrir todas las destrezas lingüísticas (ES)

- CALL complementa la labor docente (ES)

\section{Oportunidades}

- Variedad y versatilidad de materiales en CALL (ES/IR)

- CALL aumenta el interés y la motivación del alumnado (ES/IR)

- Interactividad (ES/IR)

\section{(ES/IR)}

- La implementación de CALL es abrumadora (ES/IR)

- La implementación de CALL requiere demasiado trabajo (ES)

- Existe una falta de confianza del profesor en el uso de CALL (ES)

- CALL pone nerviosos a los docentes (IR)

\section{Amenazas}

- Fallos técnicos / mal funcionamiento / averías (ES/IR)

- Cuestiones técnicas (ES/IR)

- Falta de materiales CALL estandarizados (ES/IR)

- CALL consume mucho tiempo (ES/IR) 
- $\quad$ Flexibilidad (ES/IR)

- CALL aumenta la participación de alumnado (ES/IR)

- Fomento del aprendizaje autónomo a través de CALL (ES/IR)

- $\quad$ Eficiencia $(E S / I R)$

- $\quad$ Mayor atractivo (ES/IR)

- CALL proporciona a los estudiantes una retroalimentación instantánea e individualizada (ES/IR)

- CALL ofrece un sinfín de oportunidades (ES/IR)

- Dinamismo (ES/IR)

- CALL mantiene la atención del alumnado (ES/IR)

- CALL aumenta la autonomía del alumnado (ES/IR)

- Los docentes desarrollan diferentes estilos de aprendizaje a través de CALL (ES/IR)

- CALL disminuye el estrés del alumnado (IR)

- CALL estimula la curiosidad del alumnado (ES)

- CALL aumenta el interés por aprender (ES)

- CALL hace a los estudiantes activos (ES)

- CALL evita las inhibiciones de los
- Confusión y distracción de los estudiantes a través de CALL (ES/IR)

- Falta de infraestructura o equipos antiguos (ES/IR)

- Poco fiable (ES/IR)

- CALL disminuye la comunicación cara a cara y oral (IR)

- Existe una falta de competencia digital / en el uso de CALL por parte de los estudiantes (ES/IR)

- CALL puede sustituir a los docentes (ES)

- Equipos e instalaciones costosas (ES/IR)

- Difícil de utilizar / implementar (ES/IR)

- Las tecnologías requieren mucho mantenimiento (ES/IR)

- Malos contenidos en algunos sitios web (ES/IR)

- CALL no puede ser independiente (ES)

- $\quad$ El feedback no siempre es preciso a través de CALL (ES)

- Dependencia excesiva de los estudiantes hacia CALL (IR) 
estudiantes (IR)

- CALL aporta innovación y novedad (ES/IR)

- $\quad$ Adaptabilidad (ES)

- CALL proporciona diferentes tipos de input (ES)

- Multimodal (IR)

- CALL mejora el pensamiento crítico del estudiante (IR)

(Clave: IR: profesores de inglés como lengua extranjera de Irán; ES: profesores de inglés como lengua extranjera de España)

Fuente: Elaboración propia.

3.2. Matriz DAFO de las percepciones y actitudes del alumnado hacia CALL

En la siguiente matriz, los factores internos están relacionados directamente con los estudiantes, mientras que los factores externos tienen que ver con los profesores y la propia tecnología.

Tabla 7. Matriz DAFO del alumnado de lenguas extranjeras de Irán y de España (por frecuencia de respuesta).

\begin{tabular}{|c|c|}
\hline Fortalezas & Debilidades \\
\hline $\begin{array}{l}\text { - CALL ofrece una amplia gama de } \\
\text { herramientas, recursos y materiales } \\
\text { (IR/ES) } \\
\text { - } C A L L \text { ayuda al alumnado a aprender } \\
\text { de manera más eficiente y efectiva } \\
\text { (IR/ES) } \\
\text { - CALL mejora el aprendizaje de } \\
\text { idiomas (IR/ES) } \\
\text { - CALL proporciona comunicación } \\
\text { real con hablantes nativos (IR/ES) } \\
\text { CALL disminuye la ansiedad y el } \\
\text { estrés del alumnado (IR/ES) } \\
\text { CALL proporciona }\end{array}$ & 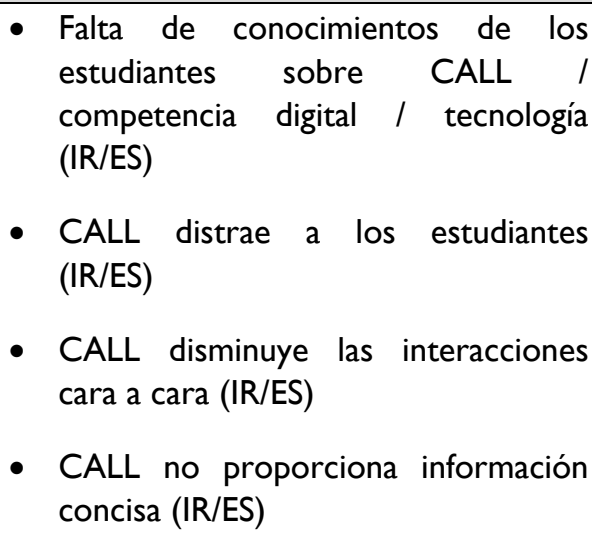 \\
\hline
\end{tabular}


retroalimentación inmediata, imparcial y constante (IR/ES)

- CALL fomenta el aprendizaje autónomo (IR/ES)

- CALL aumenta las interacciones entre pares (IR/ES)

- CALL proporciona materiales auténticos (IR/ES)

- CALL aumenta la motivación del alumnado (IR/ES)

- CALL facilita el aprendizaje (IR/ES)

- CALL aumenta la confianza del alumnado (IR)

- CALL impulsa el aprendizaje personalizado (IR/ES)

- El alumnado puede seguir su progreso a través de CALL (IR)

- CALL se adapta a diferentes estilos de aprendizaje (IR)

\section{Oportunidades}

- CALL es divertido e interesante (IR/ES)

- Aprender con CALL es cómodo y fácil (IR/ES)

- CALL es accesible y está disponible (IR/ES)

- El trabajo con CALL es rápido (IR/ES)

- Aprendizaje en cualquier momento $y$ en cualquier lugar (IR/ES)

- CALL es flexible (IR/ES)

- CALL es moderno y actual (IR/ES)

- Trabajar con CALL ahorra tiempo,
- CALL hace que los estudiantes dependan más de la tecnología (IR/ES)

- La variedad de materiales de CALL confunde a los alumnos (IR)

- Los estudiantes no se sienten seguros cuando usan CALL (IR)

- CALL no aborda todos los estilos de aprendizaje (ES)
- Dificultades técnicas (IR/ES)

- Fallos en CALL (IR/ES)

- Falta de instalaciones e infraestructura (IR/ES)

- Trabajar con CALL lleva mucho tiempo (IR/ES)

- CALL es caro (IR)

- CALL es aburrido (IR)

- CALL no siempre está disponible (IR)

- Efectos negativos sobre la salud (IR/ES)

- Contenidos de baja calidad (IR/ES)

- CALL dificulta el papel de los 
dinero y energía (IR/ES)

- CALL es preciso (IR)

- CALL es interactivo (IR/ES)

- CALL es atractivo (IR/ES)

- CALL es fácil de usar (IR/ES) docentes (IR/ES)

- CALL no proporciona suficientes directrices (IR)

- Falta de competencia de los docentes en CALL / informática /tecnología (IR)

- CALL es complejo y no es fácil de usar (ES)

- CALL no es fiable (IR)

(Clave: IR: estudiantes de inglés como lengua extranjera de Irán; ES: estudiantes de inglés como lengua extranjera de España)

Fuente: Elaboración propia.

3.3. Matriz DAFO de las similitudes en percepciones y actitudes de profesorado y alumnado

A continuación se recoge una matriz DAFO global que recoge las similitudes en las percepciones $y$ actitudes del profesorado y del alumnado de inglés como lengua extranjera en los dos países objeto de estudio (Irán y España).

Tabla 8. Matriz DAFO global

\begin{tabular}{|c|c|}
\hline Fortalezas & Debilidades \\
\hline $\begin{array}{l}\text { - CALL mejora el aprendizaje de } \\
\text { idiomas al cubrir todas las destrezas } \\
\text { (IR/ES) } \\
\text { - CALL fomenta el aprendizaje } \\
\text { autónomo (IR/ES) } \\
\text { CALL aumenta las interacciones } \\
\text { entre pares (IR/ES) } \\
\text { CALL proporciona materiales } \\
\text { auténticos y situaciones de } \\
\text { comunicación reales (IR/ES) } \\
\text { CALL facilita el aprendizaje (IR/ES) } \\
\text { CALL impulsa el aprendizaje } \\
\text { personalizado (IR/ES). }\end{array}$ & $\begin{array}{l}\text { - } \begin{array}{l}\text { Falta de competencia digital tanto de } \\
\text { docentes como de estudiantes } \\
\text { (IR/ES) }\end{array} \\
\text { - La implementación de CALL es } \\
\text { abrumadora, tanto para el } \\
\text { profesorado (requiere demasiado } \\
\text { trabajo (ES)) y hace que el alumnado } \\
\text { no se sienta seguro cuando usan } \\
\text { CALL (IR) } \\
\text { CALL no aborda todos los estilos de } \\
\text { aprendizaje (ES) }\end{array}$ \\
\hline Oportunidades & Amenazas \\
\hline
\end{tabular}


- CALL aumenta el interés y la motivación del alumnado (ES/IR), mejorando la calidad de la docencia

- Flexibilidad, eficiencia, dinamismo y rapidez (IR/ES)

- Aprendizaje en cualquier momento $y$ en cualquier lugar (IR/ES)

- CALL es interactivo (IR/ES) y proporciona a los estudiantes una retroalimentación instantánea e individualizada (ES/IR)

- CALL aumenta la autonomía del alumnado (ES/IR)
- Fallos técnicos / mal funcionamiento / averías (ES/IR)

- Trabajar con CALL consume mucho tiempo (IR/ES)

- Falta de alfabetización digital del alumnado y del profesorado (ES/IR)

- Equipos e instalaciones costosas (ES/IR)

- Contenidos de baja calidad en algunos sitios web (ES/IR)

- CALL no proporciona suficientes directrices (IR), de modo que puede causar confusión y distracción del alumnado (ES/IR)

(Clave: IR: participantes iraníes; ES: participantes españoles)

Fuente: Elaboración propia.

\section{Discusión y conclusión}

El propósito de este estudio intercultural ha sido explorar las fortalezas, oportunidades, debilidades y amenazas del aprendizaje de lenguas asistido por ordenador (CALL) basado en las actitudes y percepciones de profesorado y estudiantes de inglés como lengua extranjera en Irán y España, a fin de ver las similitudes y diferencias existentes entre ambos contextos y grupos de participantes, teniendo en cuenta que las actitudes y percepciones son los primeros determinantes de la intención individual de actuar (Rababah y Rababah, 2017). Para cumplir con el final del estudio, los investigadores aplicaron la metodología Delphi, el análisis DAFO y el análisis de contenido.

Los resultados revelaron que entre los diferentes factores en la categoría de fortalezas podemos destacar: (I) la mejora el aprendizaje de idiomas al cubrir todas las destrezas lingüísticas, (2) el fomento del aprendizaje autónomo, (3) el aumento de las interacciones entre pares y (4) el uso de materiales auténticos y situaciones de comunicación reales. Así mismo, resultan oportunidades destacables: (I) aumento del interés y la motivación del alumnado mejorando así la calidad de la docencia, (2) su flexibilidad, eficiencia, dinamismo y rapidez, (3) el aprendizaje en cualquier momento y en cualquier lugar, (4) su carácter interactivo y (5) el 
aumento de la autonomía del alumnado. Por otra parte, entre las debilidades señalamos: (I) la falta de competencia digital tanto de docentes como de estudiantes, (2) el hecho de que la implementación de CALL es abrumadora y que (3) CALL no aborda todos los estilos de aprendizaje. Finalmente, en relación con las amenazas, los participantes coinciden en: (I) la abundancia de fallos técnicos, (2) el consumo de tiempo, (3) la falta de alfabetización digital del alumnado y del profesorado, (4) el coste de equipos e instalaciones, y (5) la baja calidad de contenidos CALL en algunos sitios web.

Este estudio sugiere que la implementación efectiva de CALL en la enseñanza y el aprendizaje de lenguas se ve obstaculizada por una serie de factores clave que afectan tanto a docentes como a alumnos. Así, y de acuerdo con los resultados obtenidos, se puede señalar una serie de consideraciones sobre la implementación de CALL en la enseñanza de lenguas extranjeras, entre las que destacamos: (I) desarrollar más programas de formación para mejorar la competencia digital de profesorado y alumnado; (2) mejorar la dimensión cognitiva de profesorado y alumnado para cuestiones como la falta de confianza $\circ$ el nerviosismo, entre otros.; (3) desarrollar y distribuir de materiales estandarizados de CALL de acuerdo con estándares de calidad; y (4) animar a las instituciones a realizar una evaluación adecuada sobre la integración de las herramientas TIC en los procesos de enseñanza-aprendizaje.

\section{Referencias bibliográficas}

Eubanks, J-F., Yeh, H-T., \& Tseng, H. (20I8). Learning Chinese through a twentyfirst century writing workshop with the integration of mobile technology in a language immersion elementary school. Computer Assisted Language Learning, 3 I (4), 346-366.

Golshan, N., \& Tafazoli, D. (20/4). Technology-enhanced language learning tools in Iranian EFL context: Frequencies, attitudes and challenges. Procedia- Social and Behavioral Sciences, I36, II4-II8.

Hamid, S., Waycott, J., Kurnia, S., \& Chang, S. (2015). Understanding students' perceptions of the benefits of online social networking use for teaching and learning. Internet and Higher Education, 26, I-9.

Heflin, H., Shewmaker, J., \& Nguyen, J. (2017). Impact of mobile technology on student attitudes, engagement, and learning. Computers \& Education, 107, 91-99.

Henry, M., Carroll, F., Cunliffe, D., \& Kop, R. (2018). Learning a minority language through authentic conversation using an online social learning method. Computer Assisted Language Learning, 3 I (4), 32 I-345. 
Horvat, A., Dobrota, M., Krsmanovic, M., \& Cudanov, M. (20/3). Student perception of Moodle learning management system: a satisfaction and significance analysis. Interactive Learning Environments, 23(4), 515-527.

Hubbard, P., \& Levy, M. (2006). The scope of CALL education. In P. Hubbard \& M. Levy (Eds.), Teacher education in CALL (Pp. 2-20). Philadelphia, PA: John Benjamins Publishing Company.

Jin, L. (2018). Digital affordances on WeChat: learning Chinese as a second language. Computer Assisted Language Learning, 3 I (I-2), 27-52.

Kassen, M. A., Lavine, R. Z., Murphy-Judy, K., \& Peters, M. (2007). Preparing and developing technology-proficient L2 teachers. San Marcos, TX: CALICO.

Keeney, S., Hasson, F., \& McKenna, H. P. (200I). A critical review of the Delphi technique as a research methodology for nursing. International Journal of Nursing Studies, 38(2), 195-200.

Kessler, G. (2010). When they talk about CALL: Discourse in a required CALL class. CALICO Journal, 27, 376-392.

Krippendorf, K. (1980). Content analysis: An introduction to its methodologies. Londres: SAGE.

Kung, F-W. (2018). Assessing an innovative advanced academic writing course through blog-assisted language learning: Issues and resolutions. Innovations in Education and Teaching International, 55(3), 348-356.

Lam, Y. (2000). Technophilia vs. technophobia: A preliminary look at why secondlanguage teachers do or do not use technology in their classrooms. Canadian Modern Language Review, 56, 389-420.

Lin, C-H., Warschauer, M., \& Blake, R. (2016). Language learning through social networks: perceptions and reality. Language learning \& technology, 20 (I), I24-174.

Lintunen, P., Mutta, M., \& Pelttari, S. (2017). Profiling language learners in hybrid learning contexts: Learners' perceptions. The EUROCALL Review, 25(I), 6I75.

Mackey, A. \& Gass, S. M. (2005). Second language research: Methodology and design. Mahwah, NJ: Lawrence Erlbaum Associates.

Matsumoto, D. \& Yoo, S. H. (2006). Toward a new generation of cross-cultural research. Perspectives on Psychological Science, I (3), 234-250.

Mumtaz, S. (2000). Factors affecting teachers' use of information and communications technology: A review of the literature. Journal of Information Technology for Teacher Education, 9, 319-341.

Ozdamli, F., \& Uzunboylu, H. (2015). M-learning adequacy and perceptions of students and teachers in secondary schools. British Journal of Educational Technology, 46(I), I59-172. 
Phellas, C. N., Bloch, A., \& Seale, C. (20II). Structured methods: interviews, questionnaires and observation. En C. Seale (Ed.) Researching society and culture, 3rd edition (pp. 18I-205). Londres: Sage.

Pinto-Llorente, A. M., Sánchez-Gómez, M. C., García-Peñalvo, F. J., \& CasillasMartín, S. (2016). Students' perceptions and attitudes towards asynchronous technological tools in blended-learning training to improve grammatical competence in English as a second language. Computers in Human Behavior, 72, 632-643.

Rababah, I., \& Rababah, L. (2017). Investigating Arabic to Speakers of Other Languages (ASOL) Lecturers' Attitudes towards Utilizing Flipped Classroom Instruction (FCl): A Qualitative Study at Jordanian Public Universities. International Education Studies, 10(7), 94-104. doi: https://doi.org//0.5539/ies.v10n7p94

Riemer, V., \& Schrader. C. (2015). Learning with quizzes, simulations, and adventures: Students' attitudes, perceptions and intentions to learn with different types of serious games, Computers \& Education, 88, 160-168.

Schulze, M., \& Scholz, K. (2018). Learning trajectories and the role of online courses in a language program. Computer Assisted Language Learning, 3 I (3), 185-205.

Son, J. B., Robb, T., \& Charismiadji, I. (20I I). Computer literacy and competency: A survey of Indonesian teachers of English as a foreign language. CALL-EJ, I2, 26-42.

Stigler, J. \& Hiebert, J. (1999). The Teaching Gap. New York, NY: The Free Press.

Tafazoli, D. (2015). Review of computer-assisted language learning: History, merits \& barriers. In C. Coombe \& R. Khan (Eds.) Best Practice in ELT: Voices from the Classroom. (Pp. 255-265). Dubai, UAE: TESOL Arabia.

Tafazoli, D., Gómez Parra, M. E., \& Huertas Abril, C. A. (2018). A cross-cultural study on the attitudes of English language students towards computerassisted language learning. Teaching English with Technology, 18(2), 34-68.

Thamrin, H., \& Pamungkas, E. W. (2017). A rule based SWOT analysis application: A case study for Indonesian higher education institution. Procedia Computer Science, II6, I44-150.

Weber, R. P. (1990). Basic content analysis (2 ${ }^{\text {nd }}$ ed.). Newbury Park, CA: Sage.

Wright, B. M. (2017). Blended learning: Student perception of face-to-face and online EFL lessons. Indonesian Journal of Applied Linguistics, 7(I), 64-7I.

Yang, Y-F. (2018). New language knowledge construction through indirect feedback in web-based collaborative writing. Computer Assisted Language Learning, 3 I (4), 459-480. 

